\title{
Preoperative Versus Postoperative Transarterial Chemoembolization on Prognosis of Large Hepatocellular Carcinoma
}

\author{
Xiaohui Wang1,2\#, Yu Yuan#, Juncheng Wang1,2, Zishan Liư ${ }^{4}$, Minshan Chen ${ }^{1,2}$, Qunfang Zhou ${ }^{\llbracket}$, \\ Zhongguo Zhou ${ }^{1,2}$ \\ 1. Collaborative Innovation Center for Cancer Medicine, State Key Laboratory of Oncology in South China, Sun Yat-Sen University Cancer Center, \\ Guangzhou, Guangdong, 510060, P. R. China \\ 2. Department of Liver Surgery, Sun Yat-Sen University Cancer Center, Guangzhou, Guangdong, 510060, P. R. China. \\ 3. Department of Pulmonary and Critical Care Medicine, The Second Xiangya Hospital, Central South University, 139 Renmin Middle Road, Changsha, \\ Hunan 410011, China. \\ 4. Department of Minimally Invasive Interventional Radiology, and Department of Radiology, the Second Affiliated Hospital of Guangzhou Medical \\ University, Guangzhou, 510260, China.
}

\#These authors contributed equally to this work.

$\bowtie$ Corresponding authors: Qunfang Zhou: zhouqun988509@163.com; Zhong-Guo Zhou: zhouzhg@sysucc.org.cn; Tel: +86-020-8734-3115

(0) The author(s). This is an open access article distributed under the terms of the Creative Commons Attribution License (https://creativecommons.org/licenses/by/4.0/). See http://ivyspring.com/terms for full terms and conditions.

Received: 2020.11.12; Accepted: 2021.06.08; Published: 2021.08.28

\begin{abstract}
Background: Transarterial chemoembolization (TACE) has proven to be an effective adjuvant therapy with liver resection (LR) to treat patients with hepatocellular carcinoma (HCC). The aim of this study was to evaluate outcomes in patients with $\mathrm{HCC}$ larger than $5 \mathrm{~cm}$, comparing those who had TACE before LR to those who had TACE after LR.

Materials and methods: A total of 320 consecutive patients who underwent LR in combination with TACE for HCC larger than $5 \mathrm{~cm}$ from January 2009 to December 2014 were enrolled in study. Patients were divided into two groups: preoperative TACE group $(n=199)$ and postoperative TACE group $(n=121)$. Overall survival (OS) and recurrence-free survival (RFS) of patients were compared between preoperative TACE and postoperative TACE groups by propensity score-matching (PSM). We determined prognostic factors for recurrence and death using multivariate cox regression analysis.

Results: Among the 320 patients, the median age was 48 (range, 18 to 75) years, and 285 (89.1\%) patients were male. During the follow- up period, 88 (44.2\%) patients in the preoperative TACE group and $69(57.0 \%)$ patients in the postoperative TACE group died. Before PSM, both OS and RFS were significantly longer in the preoperative TACE group than those in the postoperative TACE group $(P=0.001$ and $P<0.001$, respectively). After PSM, compared to those received postoperative TACE, patients with preoperative TACE had significantly better OS (Hazard ratio $[H R]=1.92 ; 95 \%$ confidence interval $[\mathrm{Cl}], 1.22-3.02 ; P=0.005)$ and $\mathrm{RFS}(\mathrm{HR}=1.64 ; 95 \% \mathrm{Cl}, 1.16-2.32 ; P=0.005)$.

Conclusions: Patients with large HCC undergoing LR appear to derive greater disease control and survival benefit from a single preoperative TACE treatment than from postoperative TACE.
\end{abstract}

Key words: Large hepatocellular carcinoma; Transarterial chemoembolization; Liver resection; Prognosis; Propensity score matching

\section{Introduction}

Hepatocellular carcinoma (HCC) is the sixth most common form of cancer and the fourth most common cause of cancer-related death globally [1]. More than half of all HCC cases are diagnosed in
China, and many patients are not diagnosed until their tumors have grown to be large $(>5 \mathrm{~cm})$ or huge $(\geq 10 \mathrm{~cm})$ [2]. Currently, liver resection (LR) is the preferred method of treatment for patients with HCC 
[3]. Other treatments that are available include radiofrequency ablation (RFA), microwave ablation (MWA), cryoablation, and transarterial chemoembolization (TACE). Unfortunately, the high postsurgical recurrence rate of large HCC $(>5 \mathrm{~cm})$ has compromised the long-term survival $[4,5]$.

TACE is typically used as a locoregional or palliative therapy for HCC [6-8]. However, TACE has been used as a neoadjuvant therapy for large HCC prior to LR [7]. In addition, a systematic review has demonstrated that TACE can be combined with other modalities to improve the resectability rate for HCC [9]. Large HCC tumors are usually rich in neovascularization and frequently associated with micrometastases [10]. TACE effectively blocks tumor-feeding vessels via the hepatic artery, thereby killing foci of tumor in the areas treated [9]. Preoperative TACE therapy may also permit curative resection in some patients with large HCC who were not initially deemed to have resectable tumors [10, 11]. However, preoperative TACE therapy remains controversial. Some studies have shown that preoperative TACE does not improve the long-time survival [12, 13]. On the contrary, the use of preoperative TACE followed by LR has been shown to improve survival outcomes for some patients [14].

TACE has also been evaluated as a postoperative adjuvant therapy. Several studies have reported that postoperative TACE improved the survival of patients with HCC with portal vein tumor thrombus [15-17]. Another report focusing on patients with hepatitis B virus (HBV)-related HCC showed that those who received TACE after LR had significantly longer recurrence-free survival (RFS) and overall survival (OS) than those who had LR alone [18]. In that study, RFS was 25.7 months longer for those who received TACE after LR compared to those who did not. On subgroup analysis, postoperative TACE even provided clinical benefit to the patients in that study who had characteristics typically associated with poor prognosis, including young age, high alpha-fetoprotein (AFP) levels, cirrhosis, and other factors suggesting a high risk of recurrence (a single tumor with microvascular invasion, or multiple tumors) [19].

It remains unclear whether preoperative or postoperative TACE is more effective in prolonging survival and preventing recurrence in patients with large HCC who undergo LR. The aim of this study was to compare the OS and RFS rates of patients with large HCC $(>5 \mathrm{~cm})$ who had TACE before LR and those who had TACE after LR.

\section{Materials and methods}

\section{Patient Cohort}

We retrospectively reviewed data on consecutive patients diagnosed with large HCC $(>5 \mathrm{~cm})$ who had undergone liver resection at Sun Yat-sen University Cancer Center (SYSUCC) between January 2009 and December 2014. Patients were included if they met the following criteria: (1) 18 to 75 years of age; (2) ChildPugh grade A or B liver function; (3) patients with portal vein tumor thrombosis (PVTT) were limited in the right or the left branches of portal vein; (4) patients with multi-tumors less 3 , and the maximum size of the satellite was less than $3 \mathrm{~cm}$; (5) histopathologically confirmed HCC; (6) tumor diameter greater than $5 \mathrm{~cm}$ evaluated by imaging data (computed tomography $[\mathrm{CT}]$, magnetic resonance imaging [MRI]); (7) absence of extrahepatic metastases; (8) curative resection; (9) TACE received within 3 months before or 3 months after LR. Patients were excluded from the analysis if they: (1) had recurrent HCC; (2) non-R0 liver resection; (3) received previous systemic chemotherapy, targeted therapy with Solafenib, or RFA for HCC; (4) lost to follow-up within 90 days after LR; (5) incomplete clinical data; (6) received both preoperative and postoperative TACE therapy.

For the study, we divided the analytic cohort of patients into 2 groups (Figure 1). Those receiving preoperative TACE followed by LR were assigned to the TACE + LR group, and those receiving LR followed by adjuvant TACE were allocated to the LR + TACE group. The multidisciplinary team of physicians made the decisions about whether to utilize TACE and when to administer it. In general, TACE was offered if a curative resection might not be feasible or if the risk of recurrence was high. If preoperative TACE was performed, one time of TACE was done within one month before LR. For patients accepting the repeated TACE, the last TACE was performed within one month before LR. For postoperative TACE was performed, the fist TACE was done within 3 months after LR, provided that there was no evidence of recurrence on the CT or MRI. For some patients, a second or third TACE was undertaken if the tumor response appeared incomplete or if the risk of recurrence remained high.

This study was conducted in accordance with the Declaration of Helsinki and approved by the Ethics Committee of the Sun Yat-sen University Cancer Center (approval number: B2020-038-01, March 23, 2020).The need for patient consent was waived by the ethics committee due to the retrospective nature of the study. 


\section{Data characteristics and definitions}

We collected data for each patient about their demographic and clinicopathological characteristics, including sex, age, body mass index (BMI), tumor diameter, tumor number, macrovascular invasion (imaging data), microvascular invasion, hepatitis, cirrhosis, preoperative portal hypertension (defined as esophageal varices and/or splenomegaly on imaging data, combined with a decreased platelet count $\left[100 \times 10^{3} / \mu \mathrm{L}\right.$ or less]), intraoperative blood loss, intraoperative blood transfusion, number of TACE treatments, preoperative blood testing (including AFP, biochemical liver and renal function tests, prothrombin time [PT], and complete blood count). The platelet to lymphocyte ratio (PLR) was obtained by dividing the platelet count by the neutrophil count. The neutrophil to lymphocyte ratio (NLR) was obtained by dividing the neutrophil count by the lymphocyte count.

Overall survival (OS) was the primary endpoint for the study, and recurrence-free survival (RFS) was the secondary endpoint. OS was defined as the time from the first treatment (TACE or LR) to death or last follow-up, and RFS was defined as the time from the date of LR to tumor recurrence, death, or last follow-up (whichever came first). In addition, we defined the risk of recurrence for each patient based on preoperative imaging data and postoperative pathology. Preoperative macrovascular invasion evaluated by imaging data, and postoperative macrovascular invasion evaluated by imaging data combined with pathology. Patients were considered to be at intermediate risk of recurrence if they had a single tumor larger than $5 \mathrm{~cm}$, without microvascular invasion (MVI) or macrovascular invasion. They were considered to be at high risk of recurrence if they had a single tumor larger than $5 \mathrm{~cm}$ with microvascular invasion or macrovascular invasion, or if they had multiple tumors with at least one tumor larger than 5 $\mathrm{cm}[18,19]$.

\section{TACE technique}

TACE was performed using digital subtraction angiography guidance through the left or right hepatic artery, or directly through a tumor-feeding arterial branch when technically feasible. Hepatic artery angiography, which was performed using a 5 Fr catheter (RH or Yashiro), was used to assess the number, sizes, locations, and blood supply of target tumors. The embolization emulsion was a mixture of Epirubicin (Farmorubicin; Pharmacia, Tokyo, Japan) $30 \mathrm{mg}$ to $60 \mathrm{mg}$, Lobaplatin (Chang' an International Pharmaceutical, Hainan, China) $30 \mathrm{mg}$ to $50 \mathrm{mg}$, and Lipiodol (Laboratorie Guerbet, Aulnay-sous-Bois, France) $10 \mathrm{~mL}$ to $30 \mathrm{~mL}$, and it was infused into tumor-feeding arteries via a 2.7/2.8 Fr micro-catheter. The doses of the agents contained in the embolization emulsion were selected based on patient age, weight, comorbidity, tumor size, tumor number, and anticipated tolerance. The endpoint of the TACE procedure was reached when there was no flow in the tumor-feeding vessels.

\section{Liver resection}

Liver resection was performed by experienced surgeons. We developed a surgical plan based on tumor size, tumor location and liver function. The hepatectomy method contains anatomical resection and non-anatomical resection, and the extent was defined using the Brisbane 2000 Terminology of Liver Anatomy and Resections [20]. We applied Pringle's maneuver with cycles of clamping and unclamping times of 1 to 10 and 5 min each time, respectively, and controlled central venous pressure below $4 \mathrm{mmHg}$ during parenchyma dissection to control intraoperative bleeding. Curative resection was defined as the complete removal all tumors with clear margin confirmed by histopathology.

\section{Follow-up}

Follow-up visits were conducted at 2- or 3-month intervals for the first 18 months after LR, at 3or 4-month intervals for the next 18 months, and at 3or 6-month intervals thereafter. Each follow-up consisted of a physical examination, serum AFP, liver function and imaging examination (abdominal ultrasound, contrast-enhanced CT/MRI). If ultrasonography showed a new lesion but the AFP level was normal, contrast-enhanced ultrasonography and CT/MRI were conducted for confirmation. If two imaging findings indicated HCC, it was defined as a recurrence. Treatment options for patients with tumor recurrence included TACE, local ablation, repeat LR, sorafenib, or supportive therapy. The median follow-up period was 37 (range, 7-120) months and the date of last follow-up was September 30, 2019.

\section{Statistics}

Clinical and pathological characteristics were summarized using means with standard deviations (SD) for continuous covariates and frequencies with proportions for categorical covariates. Continuous variables were compared using the Mann-Whitney $U$ test and categorical variables were compared using either Pearson's $\chi 2$ test or Fisher's exact test. OS and RFS curves were estimated using the Kaplan-Meier method and compared by log-rank test. Univariate and multivariate Cox regression analyses were used to investigate the impact of potential prognostic factors on recurrence and death, including the impact of TACE pre- vs. post-LR on RFS and OS. Variables 
identified as significant in univariate analysis were entered into the multivariate Cox proportional hazards regression analysis. Regression results were reported as hazard ratios (HR) with $95 \%$ confidence intervals (CI), and they were estimated using the nonparametric log-rank test. All comparisons were two sided. SPSS 22.0 software (IBM Corp., Armonk, NY, USA) was used for all statistical analyses. $P<0.05$ was considered statistically significant.

\section{Propensity score matching (PSM)}

A PSM method for creating clinically comparable cohorts was used to balance the potential biases between two groups. The propensity score was estimated using a multivariate logistic regression by using variables of sex, age, BMI, tumor diameter, tumor number, macrovascular invasion, hepatitis, portal hypertension, AFP, platelet, cirrhosis, albumin, alanine aminotransferase, blood loss, blood transfusion, microvascular invasion, and number of TACE treatments. Patients were matched 1:1 using the nearest neighbor method with a caliber of 0.05 ; the matching process has been described in a previous study [10].

\section{Results}

\section{Study groups}

We identified 4,380 patients with HCC who were treated with LR during the study period, of whom 1,074 had HCC tumors larger than $5 \mathrm{~cm}$ (Figure 1). Initially, 328 patients met the criteria for our study. Among the 328 patients, 205 had TACE performed before LR and 123 had TACE performed after LR. However, 7 of these patients were lost to follow-up 3 months after LR and 1 patient expired during the postoperative period. This resulted in 199 patients in the TACE + LR group and 121 patients in the LR + TACE group. The 199 patients in the TACE + LR group received a total of 243 TACE treatments, and 39 patients underwent multiple TACE treatments. The 121 patients in the LR + TACE group received a total of 139 TACE treatments, and 18 underwent multiple TACE treatments.

\section{Demographic and clinicopathological characteristics (before PSM)}

The median age of all 320 patients was 48 (range, 18 to 75 ) years, and 285 (89.1\%) of these patients were male. Also, 113 (35.3\%) of the patients had tumor diameter of $10 \mathrm{~cm}$ or larger, $95(29.7 \%)$ had multiple tumors, $64(20.0 \%)$ had macrovascular invasion, 33 $(10.3 \%)$ had comorbidity, $256(80.0 \%)$ had hepatitis, 25 (7.8\%) had portal hypertension, and 159 (49.7\%) had cirrhosis (Table 1).

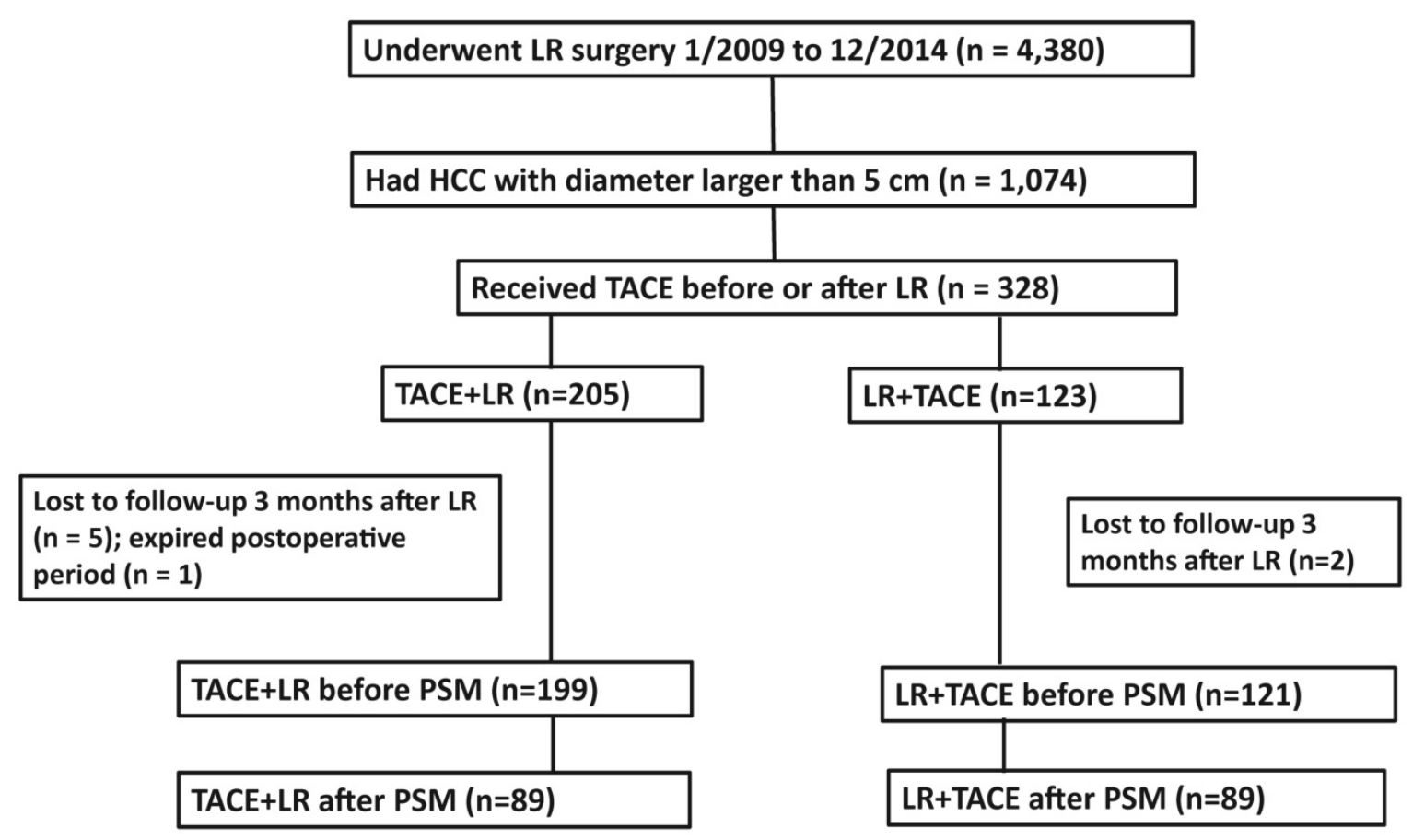

Figure 1. Flowchart of patient selection. Abbreviations: LR, liver resection; HCC, hepatocellular carcinoma; TACE, transarterial chemoembolization; PSM, propensity score matching. 
Table 1. Baseline characteristics of patients who underwent TACE + LR or LR + TACE for large hepatocellular carcinoma (HCC) before and after propensity score matchinga

\begin{tabular}{|c|c|c|c|c|c|c|}
\hline \multirow[t]{2}{*}{ Characteristics } & \multicolumn{3}{|l|}{ Before Matching } & \multicolumn{3}{|l|}{ After Matching (1:1) } \\
\hline & TACE + LR (n=199) & LR + TACE $(n=121)$ & $P$ value & TACE + LR $(n=89)$ & LR + TACE $(n=89)$ & $P$ value \\
\hline \multicolumn{7}{|c|}{ 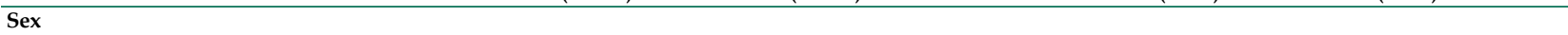 } \\
\hline Female & 25 (12.6) & $10(8.3)$ & 0.23 & $14(15.7)$ & $9(10.1)$ & 0.26 \\
\hline Male & $174(87.4)$ & $111(91.7)$ & & $75(84.3)$ & $80(89.9)$ & \\
\hline \multicolumn{7}{|l|}{ Age, years } \\
\hline$<60$ & $158(79.4)$ & $98(81.0)$ & 0.73 & $73(82.0)$ & $70(78.7)$ & 0.57 \\
\hline$\geq 60$ & $41(20.6)$ & $23(19.0)$ & & $16(18.0)$ & $19(12.3)$ & \\
\hline \multicolumn{7}{|l|}{ Child-Pugh gradeb } \\
\hline A & $190(95.5)$ & $117(96.7)$ & 0.59 & $86(96.6)$ & $87(97.8)$ & 0.65 \\
\hline B & $9(4.5)$ & $4(3.3)$ & & $3(3.4)$ & $2(2.2)$ & \\
\hline \multicolumn{7}{|l|}{ Tumor diameter, $\mathrm{cm}$} \\
\hline$<10$ & $121(60.8)$ & $86(70.5)$ & 0.06 & $63(70.8)$ & $57(64)$ & 0.34 \\
\hline$\geq 10$ & $78(39.2)$ & $35(29.5)$ & & $26(29.2)$ & $32(36)$ & \\
\hline \multicolumn{7}{|l|}{ Tumor number } \\
\hline Single & $131(65.8)$ & $94(77.7)$ & 0.02 & $65(73.0)$ & $63(70.8)$ & 0.73 \\
\hline Multiple & $68(34.2)$ & $27(22.3)$ & & $24(27.0)$ & $26(29.2)$ & \\
\hline Macrovascular invasionc & 35 (17.6) & $28(23.0)$ & 0.23 & $19(21.3)$ & $26(29.2)$ & 0.23 \\
\hline Comorbidity $^{\mathrm{d}}$ & $24(12.1)$ & $9(7.4)$ & 0.19 & $5(5.6)$ & $7(7.9)$ & 0.55 \\
\hline Hepatitise & $174(87.4)$ & $82(67.2)$ & $<0.001$ & $67(75.3)$ & $66(74.2)$ & 1.00 \\
\hline Portal hypertension ${ }^{\mathrm{f}}$ & $14(7.0)$ & $11(9.1)$ & 0.51 & $12(13.5)$ & $9(10.1)$ & 0.49 \\
\hline \multicolumn{7}{|l|}{ Alpha-fetoprotein, $\mathrm{ng} / \mathrm{mL}$} \\
\hline$\leq 400$ & $118(59.3)$ & $47(38.8)$ & $<0.001$ & $46(51.7)$ & $36(40.4)$ & 0.13 \\
\hline$>400$ & $81(40.7)$ & $74(61.2)$ & & $43(48.3)$ & $53(59.6)$ & \\
\hline \multicolumn{7}{|l|}{ Neutrophil-Lymphocyte Ratio } \\
\hline$\leq 2.8$ & 99 (49.7) & $61(50.4)$ & 0.91 & $45(50.6)$ & $46(51.7)$ & 0.88 \\
\hline$>2.8$ & $100(50.3)$ & $60(49.6)$ & & $44(49.4)$ & $43(48.3)$ & \\
\hline \multicolumn{7}{|l|}{ Platelet-Lymphocyte Ratio } \\
\hline$\leq 97$ & $67(33.7)$ & $43(35.5)$ & 0.73 & $29(32.6)$ & $35(39.3)$ & 0.35 \\
\hline$>97$ & $132(66.3)$ & $78(64.5)$ & & $60(67.4)$ & $54(60.7)$ & \\
\hline \multicolumn{7}{|l|}{ Risk of recurrenceg } \\
\hline Intermediate & $84(42.2)$ & $31(25.6)$ & 0.003 & $37(41.6)$ & $26(29.2)$ & 0.08 \\
\hline High & $115(57.8)$ & $90(74.4)$ & & $52(58.4)$ & $63(70.8)$ & \\
\hline Microvascular invasion $^{\mathrm{h}}$ & $53(26.6)$ & $56(45.9)$ & $<0.001$ & $23(25.8)$ & $31(34.8)$ & 0.19 \\
\hline Cirrhosish & $102(51.3)$ & $57(47.1)$ & 0.47 & $40(44.9)$ & $31(34.8)$ & 0.17 \\
\hline \multicolumn{7}{|l|}{ Number of TACE treatments } \\
\hline Single & $160(80.4)$ & $103(85.1)$ & 0.28 & $74(83.1)$ & $80(89.9)$ & 0.40 \\
\hline Multiple & $39(19.6)$ & $18(14.9)$ & & $14(16.9)$ & $9(10.1)$ & \\
\hline Body Mass Index, $\mathrm{kg} / \mathrm{m}^{2}$ & $22.3 \pm 3.2$ & $22.3 \pm 2.7$ & 0.68 & $21.9 \pm 3.4$ & $22.1 \pm 2.7$ & 0.83 \\
\hline Prothrombin time, seconds & $12.1 \pm 1.3$ & $12.03 \pm 1.1$ & 0.57 & $12.1 \pm 1.1$ & $11.9 \pm 1.1$ & 0.58 \\
\hline Alanine aminotransferase, $\mathrm{U} / \mathrm{L}$ & $77.4 \pm 149.1$ & $62.9 \pm 79.9$ & 0.32 & $75.3 \pm 96.7$ & $67.9 \pm 90.3$ & 0.51 \\
\hline Albumin, g/L & $40.2 \pm 6.7$ & $48.0 \pm 53.7$ & 0.04 & $40.6 \pm 8.1$ & $45.8 \pm 45.6$ & 0.30 \\
\hline Total Bilirubin, $\mu \mathrm{mol} / \mathrm{L}$ & $14.2 \pm 7.2$ & $14.3 \pm 7.8$ & 0.73 & $15.1 \pm 7.7$ & $14.07 \pm 7.3$ & 0.38 \\
\hline Platelet count, $10^{9} / \mathrm{L}$ & $181 \pm 74$ & $200 \pm 78$ & 0.03 & $191 \pm 67$ & $184 \pm 67$ & 0.52 \\
\hline White Blood Count, $10^{9} / \mathrm{L}$ & $6.59 \pm 2.81$ & $6.96 \pm 2.28$ & 0.22 & $6.39 \pm 2.01$ & $6.79 \pm 2.21$ & 0.21 \\
\hline Blood loss, $\mathrm{mL}$ & $748 \pm 742$ & $622 \pm 599$ & 0.11 & $802 \pm 868$ & $680 \pm 670$ & 0.30 \\
\hline Blood transfusion, $\mathrm{mL}$ & $251 \pm 458$ & $146 . \pm 299$ & 0.03 & $256 \pm 444$ & $169 \pm 330$ & 0.14 \\
\hline
\end{tabular}

Data are $\mathrm{n}(\%)$ or mean and standard deviation.

a A propensity score matching (PSM) method for creating clinically comparable cohorts was used to correct for potential biases. Patients were matched 1:1 using the nearest neighbor method with a caliber of 0.05 .

b Child-Pugh grade is a measure of severity of liver function, based on 5 clinical factors: PT or INR, albumin, bilirubin, ascites, and hepatic encephalopathy.

c Macrovascular tumor thrombus defined as tumor located in the intrahepatic branches of the portal or hepatic veins.

d Comorbidity defined as hypertension, diabetes, coronary disease, and/or severe anemia.

e Hepatitis defined preoperatively as a history of chronic HBV infection and/or positive hepatitis C virus RNA test.

f Portal hypertension defined preoperatively as esophageal varices and/or splenomegaly on imaging studies combined with a decreased platelet count $\left[100 \times 10^{3} / \mu \mathrm{L}\right.$ or less]).

$\mathrm{g}$ Risk of recurrence defined as intermediate if patient had a single tumor larger than $5 \mathrm{~cm}$, without microvascular invasion or macrovascular tumor thrombus, and defined as if patient had a single tumor larger than $5 \mathrm{~cm}$, with microvascular invasion or macrovascular tumor thrombus, or if they had multiple tumors with at least one tumor larger than $5 \mathrm{~cm}$ [18].

$\mathrm{h}$ Histopathological findings from liver resection (LR) specimen.

Abbreviations: TACE, transarterial chemoembolization; LR, liver resection.

\section{Comparison of Groups}

Before PSM was applied, when compared to the LR + TACE group, a significantly smaller proportion of the TACE + LR group had AFP levels greater than $400 \mathrm{ng} / \mathrm{mL}(40.7 \%$ vs. $61.2 \%, P<0.001)$, high risk of recurrence $(57.8 \%$ vs. $74.4 \%, P=0.003)$, and microvascular invasion $(26.6 \%$ vs. $45.9 \%, P<0.001)$;
The TACE + LR group also had lower mean $( \pm \mathrm{SD})$ albumin $(40.2 \pm 6.8 \mathrm{~g} / \mathrm{L}$ vs. $48.1 \pm 53.7 \mathrm{~g} / \mathrm{L}, P=0.04)$ and platelet $\left(181 \pm 74 \times 10^{9} / \mathrm{L}\right.$ vs. $200 \pm 78 \times 10^{9} / \mathrm{L}, P=$ 0.03 ) levels (Table 1). Conversely, a significantly larger proportion of the TACE + LR group had multiple tumors $(34.2 \%$ vs. $22.3 \%, P=0.02)$ and hepatitis $(87.4 \%$ vs. $67.2 \%, P<0.001)$; There were no significant differences between the 2 groups for other tumor- 
related characteristics, including tumor diameter and macrovascular invasion, or for prognosis-related characteristics, including comorbidity, portal hypertension, cirrhosis, and mean intraoperative blood loss.

\section{Overall Survival (OS)}

During the follow- up period, $88(44.2 \%)$ of the patients in the TACE + LR group and $69(57.0 \%)$ of the patients in the LR + TACE group had died. Before PSM, OS rates were significantly higher in the TACE + LR group than in the LR + TACE group at 1 year $(89.0 \%$ vs. $78.2 \%, P=0.008), 2$ years $(72.0 \%$ vs. $55.2 \%$, $P=0.001), 3$ years $(59.2 \%$ vs. $44.6 \%, P=0.008)$, and 5 years $(50.3 \%$ vs. $37.3 \%, P=0.01)$ (Table 2$)$. Moreover, for the entire study period, the TACE + LR group exhibited significantly higher OS than the LR + TACE group $(P=0.004)$ (Figure $2 \mathrm{~A})$.

\section{Recurrence-free survival (RFS)}

Before PSM, the cumulative recurrence-free survival (RFS) rates were not significantly higher in the TACE + LR group than the LR + TACE group at 1 year $(47.1 \%$ vs. $39.1 \%, P=0.08)$, but they were significantly higher at 2 years $(38.3 \%$ vs. $23.1 \%, P=$ $0.003), 3$ years $(31.7 \%$ vs. $21.3 \%, P=0.02)$, and 5 years $(27.5 \%$ vs. $18.1 \%, P=0.03)$ (Table 2). The late recurrence rate (RFS > 2 years) was significantly higher in the TACE + LR group than the LR + TACE group $(P=0.001)$. Conversely, the early recurrence rate (RFS $\leq 2$ years) was lower in the TACE + LR group, but this difference was not significant $(P=$ 0.08 ). The median RFS was 10.4 (95\% CI, 8.0-12.8) months in the TACE + LR group and 8.8 (95\% CI, 5.8-11.7) months in the LR + TACE group, a difference of 1.6 months. However, for the entire study period, the difference in RFS between the 2 groups was not statistically significant $(P=0.06)$ (Figure $2 \mathrm{~B})$.

A

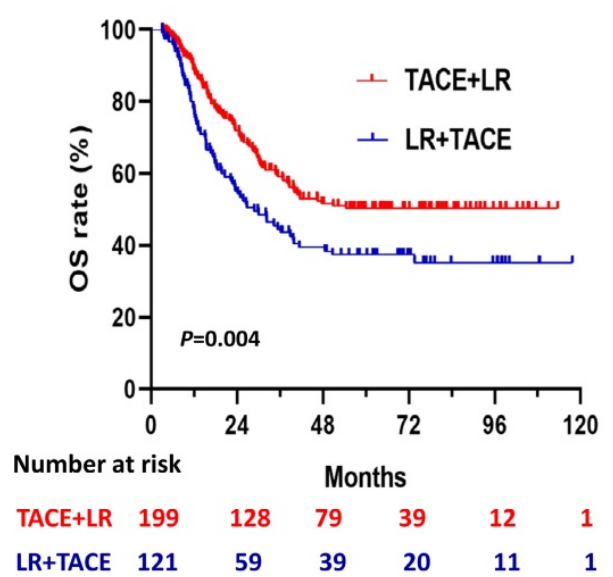

\section{Recurrence patterns}

During the follow- up period, among the 199 patients in the TACE + LR group, 148 (74.4\%) had experienced a recurrence, and of the 121 patients in the LR + TACE group $98(81.0 \%)$ had experienced a recurrence. A total of 140 of the 148 patients in the TACE + LR group and 91 of the 98 patients in the LR + TACE group had recurrence location data that we could analyze and received treatment in our department (Table 3). For the TACE + LR and the LR + TACE groups, more than half of recurrences occurred in the liver $(65.7 \%$ and $68.1 \%$, respectively), and the recurrence patterns did not differ significantly between the 2 groups $(P=0.82)$.

\section{Propensity score matching (PSM) analysis}

The 1:1 PSM analysis resulted in 89 patients each in the TACE + LR and LR + TACE groups (Table 1). It also resulted in the elimination of every significant demographic and clinicopathological characteristic difference observed between the 2 groups. After PSM, OS rates remained significantly higher in the TACE + LR group than in the LR + TACE group at 1 year $(89.4 \%$ vs. $77.4 \%, P=0.02), 2$ years $(70.9 \%$ vs. $51.9 \%, P$ $=0.005), 3$ years $(63.3 \%$ vs. $39.6 \%, P=0.001)$, and 5 years ( 53.4 vs. $32.3 \%, P=0.003$ ) (Table 2 ), and the OS rate for the entire period was also significantly higher in the TACE + LR group $(P=0.001)$ (Figure $3 \mathrm{~A})$.

After PSM, cumulative RFS rates were significantly higher in the TACE + LR group than the LR + TACE group at 1 year $(55.1 \%$ vs. $35.2 \%, P=$ $0.003), 2$ years $(44.8 \%$ vs. $17.6 \%, P<0.001), 3$ years $(37.8 \%$ vs. $17.6 \%, P=0.001)$, and 5 years $(31.4 \%$ vs. $14.7 \%, P=0.004)$ (Table 2). The RFS rate for the entire period was also significantly higher in the TACE + LR group $(P<0.001)$ (Figure 3B). The pattern of recurrence did not differ between the 2 groups, even after PSM $(P=0.34)$ (Table 3$)$.

B

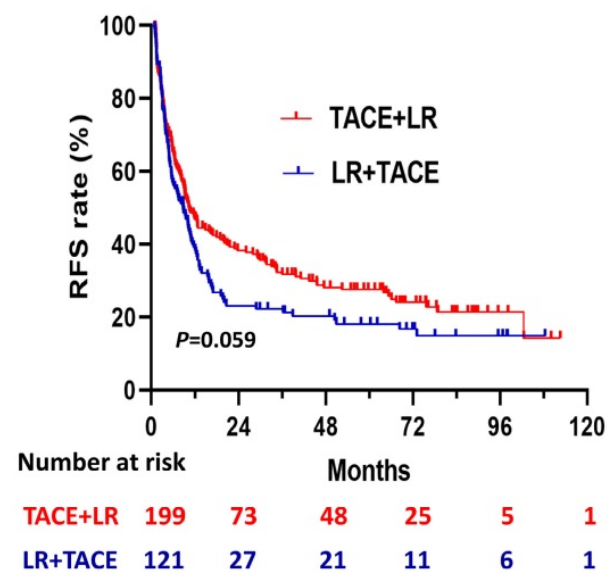

Figure 2. Kaplan-Meier curves for (A) overall survival (OS) and (B) recurrence-free survival (RFS) in patients with hepatocellular carcinoma (HCC) larger than 5 cm, comparing those who underwent transhepatic arterial chemotherapy and embolization (TACE) + LR to those who underwent LR + TACE prior to propensity score matching. 
A

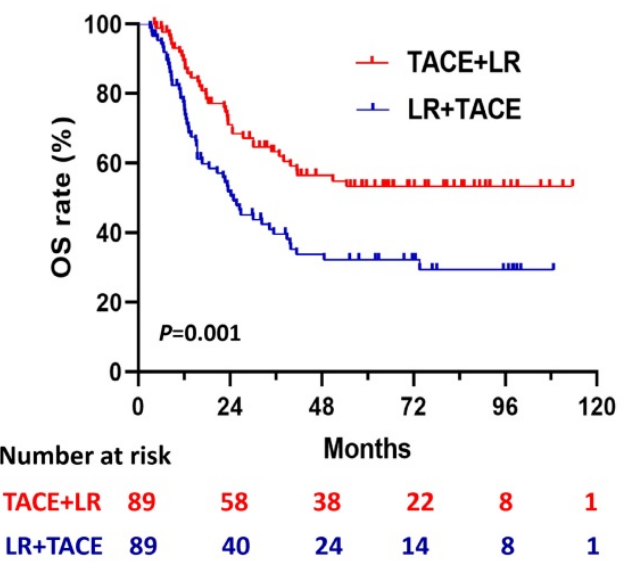

B

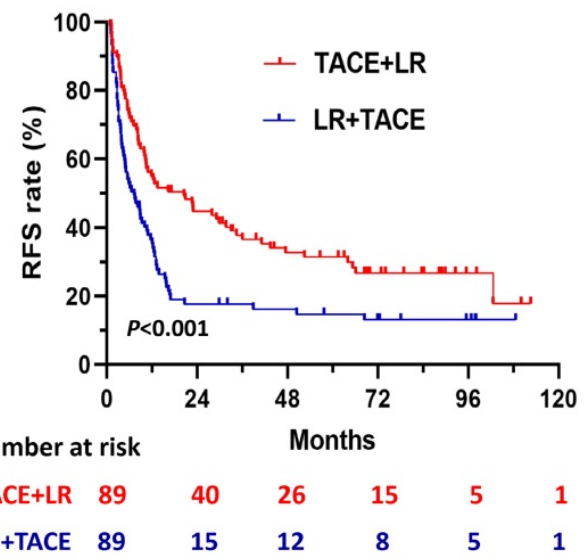

Figure 3. Kaplan-Meier curves for (A) overall survival (OS) and (B) recurrence-free survival (RFS) in patients with hepatocellular carcinoma (HCC) larger than $5 \mathrm{~cm}$, comparing those who underwent TACE + LR to those who underwent LR + TACE after propensity score matching (PSM).

Table 2. Overall survival (OS) and recurrence-free survival (RFS) of patients who underwent TACE + LR or LR + TACE for large hepatocellular carcinoma (HCC) before and after propensity score matchinga

\begin{tabular}{|c|c|c|c|c|c|c|}
\hline \multirow[t]{2}{*}{ Variables } & \multicolumn{3}{|l|}{ Before Matching } & \multicolumn{3}{|l|}{ After Matching (1:1) } \\
\hline & TACE + LR (n=199) \% & LR + TACE $(n=121) \%$ & $P$ value & TACE + LR (n=89) \% & LR + TACE (n=89) \% & $P$ value \\
\hline 1-year OS & 89.0 & 78.2 & 0.008 & 89.4 & 77.4 & 0.02 \\
\hline 2-year OS & 72.0 & 55.2 & 0.001 & 70.9 & 51.9 & 0.005 \\
\hline 3-year OS & 59.2 & 44.6 & 0.008 & 63.3 & 39.6 & 0.001 \\
\hline 5-year OS & 50.3 & 37.3 & 0.01 & 53.4 & 32.3 & 0.003 \\
\hline 1-year RFS & 47.1 & 39.1 & 0.08 & 55.1 & 35.2 & 0.003 \\
\hline 2-year RFS & 38.3 & 23.1 & 0.003 & 44.8 & 17.6 & $<0.001$ \\
\hline 3-year RFS & 31.7 & 21.3 & 0.02 & 37.8 & 17.6 & 0.001 \\
\hline 5-year RFS & 27.5 & 18.1 & 0.03 & 31.4 & 14.7 & 0.004 \\
\hline
\end{tabular}

a A propensity score matching (PSM) method for creating clinically comparable cohorts was used to correct for potential biases. Patients were matched 1:1 using the nearest neighbor method with a caliber of 0.05 .

Abbreviations: TACE, transarterial chemoembolization; LR, liver resection. OS, overall survival; RFS; recurrence-free survival

Table 3. Recurrence patterns in patients who underwent TACE + LR or LR + TACE for large hepatocellular carcinoma (HCC) before and after propensity score matchinga

\begin{tabular}{|c|c|c|c|c|c|c|}
\hline \multirow[t]{2}{*}{ Variables } & \multicolumn{3}{|l|}{ Before Matching } & \multicolumn{3}{|c|}{ After Matching (1:1) } \\
\hline & TACE+LR (n=199) & LR +TACE $(n=121)$ & P value & TACE+LR $(n=89)$ & LR+ TACE $(n=89)$ & Pvalue \\
\hline Total recurrence, $\mathrm{n}(\%)$ & $140(70.4)$ & $91(75.2)$ & & $60(67.4)$ & $69(77.5)$ & \\
\hline Recurrence patterns, n (\%) & & & 0.82 & & & 0.34 \\
\hline Intrahepatic & $92(65.7)$ & $62(68.1)$ & & $34(56.7)$ & $45(65.2)$ & \\
\hline Extrahepatic & $26(18.6)$ & $14(15.4)$ & & $14(23.3)$ & $12(17.4)$ & \\
\hline Both & 22 (15.7) & 15 (16.5) & & $12(20.0)$ & $12(17.4)$ & \\
\hline
\end{tabular}

a A propensity score matching (PSM) method for creating clinically comparable cohorts was used to correct for potential biases. Patients were matched 1:1 using the nearest neighbor method with a caliber of 0.05 .

b A total of 148 patients in the TACE + LR group and 98 patients in the LR + TACE group experienced recurrences. Of these, 140 patients in the TACE + LR group and 91 patients in the LR + TACE group had recurrence location data and received treatment in our department, so were included in this analysis.

Abbreviations: TACE, transarterial chemoembolization; LR, liver resection.

\section{Prognostic factors for OS and RFS}

The Cox-proportional hazard model was applied to the group of all patients after PSM, in order to assess the demographic and clinicopathological characteristics that were potentially related to poor survival or recurrence. The results showed that, macrovascular invasion $(\mathrm{HR}=3.98 ; 95 \% \mathrm{CI}, 2.37-6.67 ; \mathrm{P}$ $<0.001)$, microvascular invasion $(\mathrm{HR}=1.64 ; 95 \% \mathrm{CI}$, 1.04-2.58; $P=0.03)$, cirrhosis $(\mathrm{HR}=2.48 ; 95 \% \mathrm{CI}$, 1.58-3.91; $P<0.001)$, and TACE treatment after surgery $(\mathrm{HR}=1.92 ; 95 \% \mathrm{CI}, 1.22-3.02 ; P=0.005)$ were significant independent risk factors associated with poorer survival (Table 4).

Similarly, the results showed that tumor diameter $(\mathrm{HR}=1.94 ; 95 \% \mathrm{CI}, 1.31-2.88 ; P<0.001)$, tumor number $(\mathrm{HR}=1.99 ; 95 \% \mathrm{CI}, 1.35-2.93 ; P=0.001)$, macrovascular invasion $(\mathrm{HR}=3.34 ; 95 \% \mathrm{CI}, 2.23-4.99 ; P$ $<0.001)$, microvascular invasion $(\mathrm{HR}=2.24 ; 95 \% \mathrm{CI}$, 1.54-3.25; $P<0.001)$, cirrhosis (HR=1.91; 95\% CI, 1.33-2.75; $P<0.001)$, high risk of recurrence $(\mathrm{HR}=1.98$; 95\% CI, 1.16-3.34; $P=0.012)$, and TACE treatment after surgery $(\mathrm{HR}=1.64 ; 95 \% \mathrm{CI}, 1.16-2.32 ; P=0.005)$ were significant independent risk factors associated with tumor recurrence (Table 5). 
Table 4. Univariate and multivariate analyses for overall survival for large hepatocellular carcinoma patients who underwent a combination of TACE and liver resection after propensity score matchinga

\begin{tabular}{|c|c|c|c|c|c|}
\hline \multirow[t]{2}{*}{ Characteristics } & \multirow{2}{*}{$\begin{array}{l}\text { Patients in Each } \\
\text { Covariate } \\
\mathrm{N}: \mathrm{N}\end{array}$} & \multicolumn{2}{|l|}{ Univariate Analysis } & \multicolumn{2}{|l|}{ Multivariate Analysis } \\
\hline & & HR (95\% CI) & P value & HR $(95 \%$ CI) & P value \\
\hline Sex (female vs. male) & $23: 155$ & $0.91(0.50-1.68)$ & 0.77 & & \\
\hline Age, years $(\geq 60$ vs. $<60)$ & $35: 143$ & $0.94(0.56-1.57)$ & 0.80 & & \\
\hline Tumor diameter $(\geq 10$ vs. $<10), \mathrm{cm}$ & $58: 120$ & $1.75(1.45-2.67)$ & 0.009 & & 0.42 \\
\hline Tumor number (multiple vs. single ) & $50: 128$ & $1.44(0.92-2.25)$ & 0.11 & & \\
\hline $\begin{array}{l}\text { Macrovascular invasion }{ }^{\mathrm{b}} \text { (positive vs. } \\
\text { negative) }\end{array}$ & $45: 143$ & $5.80(3.70-9.10)$ & $<0.001$ & $3.98(2.37-6.67)$ & $<0.001$ \\
\hline Comorbidity ${ }^{\mathrm{c}}$ (yes vs. no) & $12: 166$ & $0.49(0.18-1.33)$ & 0.16 & & \\
\hline Portal hypertension $^{\mathrm{d}}$ (yes vs. no) & $157: 21$ & $1.28(0.73-2.27)$ & 0.39 & & \\
\hline Alpha-fetoprotein, ng/mL (> 400 vs. $\leq 400)$ & $96: 82$ & $1.54(1.01-2.34)$ & 0.046 & & 0.72 \\
\hline $\begin{array}{l}\text { Neutrophil-Lymphocyte Ratio (> } 2.8 \text { vs. } \leq \\
2.8 \text { ) }\end{array}$ & $87: 91$ & $1.31(0.87-1.99)$ & 0.20 & & \\
\hline Platelet-Lymphocyte Ratio (> 97 vs. $\leq 97$ ) & $114: 64$ & $0.99(0.65-1.53)$ & -- & & \\
\hline $\begin{array}{l}\text { Microvascular invasione (positive vs. } \\
\text { negative) }\end{array}$ & $54: 124$ & $1.64(1.04-2.58)$ & 0.002 & $1.64(1.04-2.58)$ & 0.03 \\
\hline Hepatitis (yes vs. no) & 133:65 & $1.49(0.89-2.51)$ & 0.126 & & \\
\hline Cirrhosise (yes vs. no) & 71: 107 & $3.05(2.01-4.64)$ & $<0.001$ & $2.48(1.58-3.91)$ & $<0.001$ \\
\hline Number of TACE (multiple vs. single) & $26: 152$ & $1.03(0.60-1.76)$ & 0.93 & & \\
\hline Risk of recurrence (high vs. intermediate) & $99: 79$ & $3.65(2.32-5.74)$ & $<0.001$ & $1.72(0.92-3.12)$ & 0.093 \\
\hline Time of TACE (post vs. pre-resection) & $89: 89$ & $1.97(1.30-3.00)$ & 0.002 & $1.92(1.22-3.02)$ & 0.005 \\
\hline
\end{tabular}

a A propensity score matching (PSM) method for creating clinically comparable cohorts was used to correct for potential biases. Patients were matched 1:1 using the nearest neighbor method with a caliber of 0.05 .

${ }^{\mathrm{b}}$ Macrovascular tumor thrombus defined as tumor located in the intrahepatic branches of the portal or hepatic veins.

cComorbidity defined as hypertension, diabetes, coronary disease, and/or severe anemia.

d Portal hypertension defined preoperatively as esophageal varices and/or splenomegaly on imaging studies combined with a decreased platelet count $\left[100 \times 10^{3} / \mu \mathrm{L}\right.$ or less]).

e Histopathological findings from liver resection (LR) specimen.

Abbreviations: TACE, transarterial chemoembolization; HR, hazard ratio; CI, confidence interval

Table 5. Univariate and multivariate analyses for recurrence for large hepatocellular carcinoma patients who underwent a combination of TACE and hepatic resection after propensity score matchinga

\begin{tabular}{|c|c|c|c|c|c|}
\hline \multirow[t]{2}{*}{ Characteristics } & \multirow{2}{*}{$\begin{array}{l}\text { Patients in each } \\
\text { Covariate } \\
\mathrm{N}: \mathrm{N}\end{array}$} & \multicolumn{2}{|l|}{ Univariate Analysis } & \multicolumn{2}{|c|}{ Multivariate Analysis } \\
\hline & & HR $(95 \% \mathrm{CI})$ & Pvalue & HR $(95 \%$ CI) & P value \\
\hline Sex (female vs. male) & $23: 155$ & $0.78(0.47-1.33)$ & 0.37 & & \\
\hline Age, years $(\geq 60$ vs. $<60)$ & $35: 143$ & $0.93(0.61-1.41)$ & 0.73 & & \\
\hline Tumor diameter $(\geq 10$ vs. $<10), \mathrm{cm}$ & $58: 120$ & $2.62(1.84-3.73)$ & $<0.001$ & $1.94(1.31-2.88)$ & $<0.001$ \\
\hline Tumor number (multiple vs. single ) & $50: 128$ & $1.56(1.08-2.25)$ & 0.02 & $1.99(1.35-2.93)$ & 0.001 \\
\hline Macrovascular invasion ${ }^{\mathrm{b}}$ (positive vs. negative) & $45: 143$ & $4.42(2.99-6.55)$ & $<0.001$ & $3.34(2.23-4.99)$ & $<0.001$ \\
\hline Comorbidityc (yes vs. no) & $12: 166$ & $0.79(0.40-1.56)$ & 0.50 & & \\
\hline Portal hypertension ${ }^{\mathrm{d}}$ (yes vs. no) & $157: 21$ & $1.02(0.61-1.70)$ & 0.94 & & \\
\hline Alpha-fetoprotein, ng/mL (> 400 vs. $\leq 400)$ & $96: 82$ & $1.26(0.90-1.76)$ & 0.18 & & \\
\hline Neutrophil-Lymphocyte Ratio (> 2.8 vs. $\leq 2.8$ ) & $87: 91$ & $1.24(0.89-1.73)$ & 0.21 & & \\
\hline Platelet-Lymphocyte Ratio (> 97 vs. $\leq 97$ ) & $114: 64$ & $0.86(0.61-1.23)$ & 0.41 & & \\
\hline Microvascular invasione (positive vs. negative) & $54: 124$ & $2.71(1.88-3.92)$ & $<0.001$ & $2.24(1.54-3.25)$ & $<0.001$ \\
\hline Hepatitis (yes vs. no) & 133:65 & $1.1 .4(0.78-1.68)$ & 0.502 & & \\
\hline Cirrhosise (yes vs. no) & 71: 107 & $2.05(1.46-2.89)$ & $<0.001$ & $1.91(1.33-2.75)$ & $<0.001$ \\
\hline Number of TACE (multiple vs. single) & $26: 152$ & $0.94(0.59-1.50)$ & 0.79 & & \\
\hline Risk of recurrence (high vs. intermediate) & 99:79 & $4.21(2.87-6.18)$ & $<0.001$ & $1.98(1.16-3.34)$ & 0.012 \\
\hline Time of TACE (post vs. pre-resection) & $89: 89$ & $1.78(1.27-2.49)$ & 0.001 & $1.64(1.16-2.32)$ & 0.005 \\
\hline
\end{tabular}

a A propensity score matching (PSM) method for creating clinically comparable cohorts was used to correct for potential biases. Patients were matched 1:1 using the nearest neighbor method with a caliber of 0.05 .

${ }^{b}$ Macrovascular tumor thrombus defined as tumor located in the intrahepatic branches of the portal or hepatic veins.

c Comorbidity defined as hypertension, diabetes, coronary disease, and/or severe anemia.

d Portal hypertension defined preoperatively as esophageal varices and/or splenomegaly on imaging studies combined with a decreased platelet count $\left[100 \times 10^{3} / \mu \mathrm{L}\right.$ or less]).

e Histopathological findings from liver resection (LR) specimen.

Abbreviations: TACE, transarterial chemoembolization; HR, hazard ratio; $\mathrm{CI}$, confidence interval

\section{Discussion}

Tumor recurrence after LR continues to be a substantial challenge in the clinical management of large HCC, and it is associated with poor prognosis [21]. TACE is a minimally invasive therapy that has demonstrated some efficacy in the treatment of large HCC [22]. TACE inhibits tumor growth by blocking tumor blood supply and creating hypoxia which induces tumor necrosis. A number of studies have shown that TACE can improve survival outcomes in patients with unresectable HCC [23-25]. Other studies 
have also demonstrated that patients with large HCC can benefit from preoperative TACE $[26,27]$. On the other hand, previous studies have suggested that postoperative TACE can improve the prognosis of patients with a high risk of HCC recurrence after LR $[14,28]$. However, whether preoperative or postoperative TACE is more effective in prolonging survival in patients with large HCC who undergo LR is still unknown. In this study, we found that the OS rates for patients who had TACE before LR were significantly better than for those who had TACE after LR. Furthermore, a comparison of the results in our patients who received TACE prior to LR with the patients in other studies who received only LR suggests a potential benefit from the addition of TACE prior to LR $[29,30]$. We also found that the RFS rates for patients who had TACE before LR were better than those who had TACE after LR. This suggests that the addition of TACE prior to LR may possibly provide some protection against recurrence of HCC in this patient population.

Considering there are several clinicopathological characteristics that differed significantly between the 2 groups in our study, we applied PSM to the groups and repeated the comparisons, with the goal of limiting the bias inherent in the groups being different. However, even after PSM, the cumulative OS and RFS rates for the entire study period were also significantly higher in the TACE + LR group than in the LR + TACE group. In addition, the administration of preoperative TACE, as opposed to postoperative TACE, resulted in OS rates that were 12 percentage points higher at 1 year and 19 percentage points higher at both 2 years and 5 years. Moreover, again after applying PSM, the use of TACE prior to rather than after LR resulted in RFS rates that were 20 percentage points higher at 1 year, 27 percentage points higher at 2 years, and 16 percentage points higher at 5 years. Therefore, even with PSM, our results show both a statistically significant and a clinically sizable relative benefit of preoperative TACE in patients with large HCC.

Using of TACE either before or after LR has the potential to lower the risk of tumor recurrence and improve the survival rates of patients with large HCC $[10,31]$. A meta-analysis systematically reviewed the published articles of preoperative and postoperative TACE for curative resection of HCC and sought to evaluate the outcomes of the two therapies, the analysis concluded that postoperative TACE offers potential benefits for curative resection of HCC when the mean tumor size is bigger than $5 \mathrm{~cm}$. However, preoperative TACE showed no evidence in improving the RFS and OS in the patients [32]. Our result was in contrary with the meta-analysis above. Even though there were significant improvements for RFS and OS in the postoperative TACE compared with preoperative TACE in that meta-analysis, there was no strict clinical research to compared the between preoperative and postoperative TACE in improving the outcomes of large HCC. In theory, postoperative TACE improve the outcomes of patients with large HCC, over LR alone, by destroying residual occult intrahepatic disease close to the tumor bed or other adjacent satellite lesions not identified by perioperative imaging or during surgery [18, 33]. However, TACE given after LR, which has already involved the removal of a large volume of liver tissue, may accelerate the deterioration of liver function, contribute to the suppression of host immunity against tumor progression, and negatively impact the regeneration of hepatocytes [34, 35]. Collectively, these processes potentially explain the inferior long-term survival rates in the patients in our study who received TACE after LR.

Some have suggested that TACE done prior to LR might result in more difficult surgery and a higher risk for intraoperative bleeding, due to the hepatic inflammation caused by TACE [36]. Conversely, others have reported that preoperative TACE seems to have little influence on subsequent surgery $[10,37]$. In our study, before PSM, the TACE + LR group did indeed have higher mean intraoperative blood loss and blood transfusion volumes than the LR + TACE group, while after PSM, neither of these differences was statistically significant. Thus, although we observed numerically higher volumes of blood loss and transfusion when LR was preceded by TACE, these differences were not statistically significant, nor were they likely clinically relevant.

In the present study, before PSM, we found that the proportion of patients with microvascular invasion identified pathology after surgery was significantly lower in the TACE + LR group than in the LR + TACE group. This is not surprising, and others have reported that preoperative TACE for HCC can induce massive necrosis, which can markedly reduce the amount of microvascular invasion in the tumor [10]. Considering that large $\mathrm{HCC}$ is often associated with extensive microvascular invasion, and that microvascular invasion is a known risk factor of poor prognosis in large $\mathrm{HCC}$, the relative reduction of microvascular invasion in patients receiving TACE prior to LR may provide one possible explanation for why those patients had better outcomes in our study [38]. Patients with large HCC also often have micrometastases, which traditional imaging modalities usually fail to detect [39]. Preoperative TACE may be able to find them and may be effective in shortening the development of 
micrometastases, by interrupting the process of local tumor microvascular invasion at an earlier point in time. This may be another possible explanation for the better long-term outcomes in patients in our study who received preoperative TACE.

In the population of our study, the significant independent predictors of poorer overall survival were macrovascular invasion, microvascular invasion, cirrhosis, and TACE treatment after surgery. The significant independent predictors of HCC recurrence were tumor diameter, multiple tumors, macrovascular invasion, microvascular invasion, cirrhosis, and TACE treatment after surgery. These findings are similar to those in other studies [10, 18]. Additionally, patients in our study having multiple TACE treatments, either before or after LR, had RFS and OS rates that did not differ significantly from the rates of those who had only a single TACE treatment. Others have reported that a single TACE treatment (which includes hepatic angiography) generally allows for the detection of any additional small HCC satellite nodules and the confirmation that local tumor vascular supply has been disrupted [36, 40]. They concluded that preoperative TACE should not be repeated and that doing so would have no significant effect on recurrence and survival. The results from our study suggest that patients with large HCC need only be treated with a single TACE treatment for maximum benefit.

This study has several limitations. First, it was a retrospective single-center study. A prospective, randomized study involving multiple centers and a more heterogeneous patient population would be necessary to confirm our findings. Second, patients in this study were not specifically evaluated for whether they had a good response to TACE prior to LR. Future studies should incorporate radiomics or biological markers to help measure the response to TACE, so that only those patients who have a good response are included in comparisons. Third, measuring the response to TACE would also be helpful clinically, because patients who did not have a good response could then be offered alternative treatments prior to $\mathrm{LR}$, such as thermal ablation.

\section{Conclusions}

Patients with large HCC undergoing LR appear to derive greater disease control and survival benefit from preoperative TACE than from postoperative TACE. A single TACE treatment appears to be sufficient; RFS and OS are not improved by the administration of multiple TACE treatments. These results provide valuable information to guide the management of patients with large HCC.

\section{Acknowledgments}

This work was funded by the National Natural Science Foundation of China (NO.81874070), Sun Yat-Sen University Cancer Center Physician-scientist Funding (16zxqk04), Guangdong Key Laboratory of Liver Disease Research (GS2017011002).

\section{Competing Interests}

The authors have declared that no competing interest exists.

\section{References}

1. Bray F, Ferlay J, Soerjomataram I, Siegel RL, Torre LA, Jemal A. Global cancer statistics 2018: GLOBOCAN estimates of incidence and mortality worldwide for 36 cancers in 185 countries. CA Cancer J Clin. 2018; 68: 394-424.

2. Chen $\mathrm{W}$, Zheng $\mathrm{R}$, Zeng $\mathrm{H}$, Zhang $\mathrm{S}$. The incidence and mortality of major cancers in China, 2012. Chin J Cancer. 2016; 35: 73.

3. Chau GY. Resection of hepatitis B virus-related hepatocellular carcinoma: evolving strategies and emerging therapies to improve outcome. World J Gastroenterol. 2014; 20: 12473-84.

4. Wakayama K, Kamiyama T, Yokoo H, Orimo T, Shimada S, Einama T, et al. Huge hepatocellular carcinoma greater than $10 \mathrm{~cm}$ in diameter worsens prognosis by causing distant recurrence after curative resection. J Surg Oncol. 2017; 115: 324-9.

5. Yang L, Xu J, Ou D, Wu W, Zeng Z. Hepatectomy for huge hepatocellular carcinoma: single institute's experience. World J Surg. 2013; 37: 2189-96.

6. Luo YQ, Wang Y, Chen $\mathrm{H}, \mathrm{Wu} \mathrm{MC}$. Influence of preoperative transcatheter arterial chemoembolization on liver resection in patients with resectable hepatocellular carcinoma. Hepatobiliary Pancreat Dis Int. 2002; 1: 523-6.

7. Yamashita Y, Takeishi K, Tsuijita E, Yoshiya S, Morita K, Kayashima H, et al. Beneficial effects of preoperative lipiodolization for resectable large hepatocellular carcinoma ( $\geq 5 \mathrm{~cm}$ in diameter). J Surg Oncol. 2012; 106: 498-503.

8. Nishikawa H, Arimoto A, Wakasa T, Kita R, Kimura T, Osaki Y. Effect of transcatheter arterial chemoembolization prior to surgical resection for hepatocellular carcinoma. Int J Oncol. 2013; 42: 151-60.

9. Tustumi F, Ernani L, Coelho FF, Bernardo WM, Junior SS, Kruger JAP, et al. Preoperative strategies to improve resectability for hepatocellular carcinoma: a systematic review and meta-analysis. HPB (Oxford). 2018; 20: 1109-18.

10. Li C, Wang MD, Lu L, Wu H, Yu JJ, Zhang WG, et al. Preoperative transcatheter arterial chemoembolization for surgical resection of huge hepatocellular carcinoma $(\geq 10 \mathrm{~cm})$ : a multicenter propensity matching analysis. Hepatol Int. 2019; 13: 736-47.

11. Choi JH, Hwang S, Lee YJ, Kim KH, Ko GY, Gwon DI, et al. Prognostic effect of preoperative sequential transcatheter arterial chemoembolization and portal vein embolization for right hepatectomy in patients with solitary hepatocellular carcinoma. Korean J Hepatobiliary Pancreat Surg. 2015; 19: $59-65$.

12. Zhou WP, Lai EC, Li AJ, Fu SY, Zhou JP, Pan ZY, et al. A prospective, randomized, controlled trial of preoperative transarterial chemoembolization for resectable large hepatocellular carcinoma. Ann Surg. 2009; 249: 195-202.

13. Yamasaki S, Hasegawa H, Kinoshita H, Furukawa M, Imaoka S, Takasaki K, et al. A prospective randomized trial of the preventive effect of pre-operative transcatheter arterial embolization against recurrence of hepatocellular carcinoma. Jpn J Cancer Res. 1996; 87: 206-11

14. Zhang YF, Guo RP, Zou RH, Shen JX, Wei W, Li SH, et al. Efficacy and safety of preoperative chemoembolization for resectable hepatocellular carcinoma with portal vein invasion: a prospective comparative study. Eur Radiol. 2016; 26: 2078-88.

15. Liu S, Guo L, Li H, Zhang B, Sun J, Zhou C, et al. Postoperative Adjuvant Trans-Arterial Chemoembolization for Patients with Hepatocellular Carcinoma and Portal Vein Tumor Thrombus. Ann Surg Oncol. 2018; 25: 2098-104.

16. Chen ZH, Zhang XP, Lu YG, Li LQ, Chen MS, Wen TF, et al. Actual long-term survival in HCC patients with portal vein tumor thrombus after liver resection: a nationwide study. Hepatol Int. 2020; 14: 754-64.

17. Gao Y, Wang PX, Cheng JW, Sun YF, Hu B, Guo W, et al. Chemotherapeutic perfusion of portal vein after tumor thrombectomy and hepatectomy benefits patients with advanced hepatocellular carcinoma: A propensity score-matched survival analysis. Cancer Med. 2019; 8: 6933-44.

18. Wang Z, Ren Z, Chen Y, Hu J, Yang G, Yu L, et al. Adjuvant Transarterial Chemoembolization for HBV-Related Hepatocellular Carcinoma After Resection: A Randomized Controlled Study. Clin Cancer Res. 2018; 24: 2074-81.

19. EASL-EORTC clinical practice guidelines: management of hepatocellular carcinoma. J Hepatol. 2012; 56: 908-43.

20. Strasberg SM. Nomenclature of hepatic anatomy and resections: a review of the Brisbane 2000 system. J Hepatobiliary Pancreat Surg. 2005; 12: 351-5. 
21. Omata M, Cheng AL, Kokudo N, Kudo M, Lee JM, Jia J, et al. Asia-Pacific clinical practice guidelines on the management of hepatocellular carcinoma: a 2017 update. Hepatol Int. 2017; 11: 317-70.

22. Wei CY, Chen PC, Chau GY, Lee RC, Chen PH, Huo TI, et al. Comparison of prognosis between surgical resection and transarterial chemoembolization for patients with solitary huge hepatocellular carcinoma. Ann Transl Med. 2020; 8: 238.

23. Lin CW, Chen YS, Lo GH, Hsu YC, Hsu CC, Wu TC, et al. Comparison of overall survival on surgical resection versus transarterial chemoembolization with or without radiofrequency ablation in intermediate stage hepatocellular carcinoma: a propensity score matching analysis. BMC Gastroenterol. 2020; 20: 99.

24. Zhao J, Zeng L, Wu Q, Wang L, Lei J, Luo H, et al. Stereotactic Body Radiotherapy Combined with Transcatheter Arterial Chemoembolization versus Stereotactic Body Radiotherapy Alone as the First-Line Treatment for Unresectable Hepatocellular Carcinoma: A Meta-Analysis and Systematic Review. Chemotherapy. 2019; 64: 248-58.

25. Chiang CL, Chan ACY, Chiu KWH, Kong FS. Combined Stereotactic Body Radiotherapy and Checkpoint Inhibition in Unresectable Hepatocellular Carcinoma: A Potential Synergistic Treatment Strategy. Front Oncol. 2019; 9: 1157.

26. Sugo H, Futagawa S, Beppu T, Fukasawa M, Kojima K. Role of preoperative transcatheter arterial chemoembolization for resectable hepatocellular carcinoma: relation between postoperative course and the pattern of tumor recurrence. World J Surg. 2003; 27: 1295-9.

27. Majno PE, Adam R, Bismuth H, Castaing D, Ariche A, Krissat J, et al. Influence of preoperative transarterial lipiodol chemoembolization on resection and transplantation for hepatocellular carcinoma in patients with cirrhosis. Ann Surg. 1997; 226: 688-701; discussion -3.

28. Chen X, Zhang B, Yin X, Ren Z, Qiu S, Zhou J. Lipiodolized transarterial chemoembolization in hepatocellular carcinoma patients after curative resection. J Cancer Res Clin Oncol. 2013; 139: 773-81.

29. Yin L, Li H, Li AJ, Lau WY, Pan ZY, Lai EC, et al. Partial hepatectomy vs. transcatheter arterial chemoembolization for resectable multiple hepatocellular carcinoma beyond Milan Criteria: a RCT. J Hepatol. 2014; 61: 82-8.

30. Hwang S, Joh JW, Wang HJ, Kim DG, Kim KS, Suh KS, et al. Prognostic Prediction Models for Resection of Large Hepatocellular Carcinoma: A Korean Multicenter Study. World J Surg. 2018; 42: 2579-91.

31. Ye JZ, Chen JZ, Li ZH, Bai T, Chen J, Zhu SL, et al. Efficacy of postoperative adjuvant transcatheter arterial chemoembolization in hepatocellular carcinoma patients with microvascular invasion. World J Gastroenterol. 2017; 23: 7415-24.

32. Cheng X, Sun P, Hu QG, Song ZF, Xiong J, Zheng QC. Transarterial (chemo)embolization for curative resection of hepatocellular carcinoma: a systematic review and meta-analyses. J Cancer Res Clin Oncol. 2014; 140: 1159-70.

33. Aufhauser $\mathrm{DD}, \mathrm{Jr}$, Sadot $\mathrm{E}$, Murken $\mathrm{DR}$, Eddinger $\mathrm{K}$, Hoteit $\mathrm{M}$, Abt PL, et al Incidence of Occult Intrahepatic Metastasis in Hepatocellular Carcinoma Treated With Transplantation Corresponds to Early Recurrence Rates After Partial Hepatectomy. Ann Surg. 2018; 267: 922-8.

34. Poon RT, Fan ST, Lo CM, Liu CL, Ng IO, Wong J. Long-term prognosis after resection of hepatocellular carcinoma associated with hepatitis B-related cirrhosis. J Clin Oncol. 2000; 18: 1094-101.

35. Huang G, Lai EC, Lau WY, Zhou WP, Shen F, Pan ZY, et al. Posthepatectomy $\mathrm{HBV}$ reactivation in hepatitis B-related hepatocellular carcinoma influences postoperative survival in patients with preoperative low HBV-DNA levels. Ann Surg. 2013; 257: 490-505.

36. Paye F, Jagot P, Vilgrain V, Farges O, Borie D, Belghiti J. Preoperative chemoembolization of hepatocellular carcinoma: a comparative study. Arch Surg. 1998; 133: 767-72

37. Nagasue N, Galizia G, Kohno $H$, Chang YC, Hayashi $T$, Yamanoi A, et al Adverse effects of preoperative hepatic artery chemoembolization for resectable hepatocellular carcinoma: a retrospective comparison of 138 liver resections. Surgery. 1989; 106: 81-6.

38. Lu CD, Peng SY, Jiang XC, Chiba Y, Tanigawa N. Preoperative transcatheter arterial chemoembolization and prognosis of patients with hepatocellular carcinomas: retrospective analysis of 120 cases. World J Surg. 1999; 23: 293-300.

39. Jin Y, Wang K, Tian J. Preoperative Examination and Intraoperative Identification of Hepatocellular Carcinoma Using a Targeted Bimodal Imaging Probe. Bioconjug Chem. 2018; 29: 1475-84.

40. Portolani N, Tiberio AM, Bonardelli S, Grazioli L, Matricardi L, Benetti A, et al. Arterial chemoembolization in hepatocellular carcinoma suitable for resective surgery. Hepatogastroenterology. 1996; 43: 1566-74. 